\title{
Tunable superconducting microstrip resonators
}

\author{
A. A. Adamyan, a) S. E. Kubatkin, and A. V. Danilov \\ Department of Microtechnology and Nanoscience, MC2, Chalmers University of Technology, \\ SE-41296 Göteborg, Sweden
}

(Received 11 February 2016; accepted 14 April 2016; published online 25 April 2016)

\begin{abstract}
We report on a simple yet versatile design for a tunable superconducting microstrip resonator. Niobium nitride is employed as the superconducting material and aluminum oxide, produced by atomic layer deposition, as the dielectric layer. We show that the high quality of the dielectric material allows to reach the internal quality factors in the order of $Q_{i} \sim 10^{4}$ in the single photon regime. $Q_{i}$ rapidly increases with the number of photons in the resonator $N$ and exceeds $10^{5}$ for $N \sim 10-50$. A straightforward modification of the basic microstrip design allows to pass a current bias through the strip and to control its kinetic inductance. We achieve a frequency tuning $\delta f=62 \mathrm{MHz}$ around $f_{0}=2.4 \mathrm{GHz}$ for a fundamental mode and $\delta f=164 \mathrm{MHz}$ for a third harmonic. This translates into a tuning parameter $Q_{i} \delta f / f_{0}=150$. The presented design can be incorporated into essentially any superconducting circuitry operating at temperatures below 2.5 K. Published by AIP Publishing. [http://dx.doi.org/10.1063/1.4947579]
\end{abstract}

Planar superconducting resonators are an enabling technology for quantum information processing, ${ }^{1}$ hybrid quantum memory systems, ${ }^{2,3}$ electron spin resonance (ESR) spectroscopy on femto mole samples, ${ }^{4}$ kinetic inductance detectors, ${ }^{5}$ and parametric amplifiers, ${ }^{6}$ among many other applications. The resonators are instrumental for the whole field of circuit quantum electrodynamics (c-QED) for having dramatically reduced microwave mode volume, as compared to bulk cavities, and accordingly increased coupling to engineered q-bits and spins. ${ }^{7,8}$

Among the two generic planar resonator types, microstrip and coplanar, both are ubiquitous in industrial applications,,${ }^{9,10}$ while only the latter is dominant in superconducting designs. This is because, in pursuit to squeeze the mode volume, the central line-to-ground gap in a typical coplanar resonator for c-QED applications is only a few microns wide. An equivalent microstrip resonator should have a strip-to-ground dielectric layer also just a few microns thick, which excludes a dielectric substrate as a spacer and calls for a multilayer design with a thin dielectric film.

Until recently, all reports on microwave losses in the deposited dielectrics indicated that these materials are discouragingly inferior to a crystalline sapphire commonly used as a substrate in coplanar waveguides (CPWs); it is only after an extended search and refined fabrication processes that a loss tangent below $10^{-4}$ was reached, ${ }^{11}$ opening for c-QED applications.

Apart from the high Q-factor, many applications benefit from a variable resonance frequency. Tuning the resonance frequency is a pre-requisite for the dynamic coupling of quantum bits (qubits), ${ }^{12}$ controllable storage and release of microwave photons ${ }^{13}$ photon generation via the dynamical Casimir effect, ${ }^{14}$ and parametric amplification. ${ }^{15,16}$ In all the above examples, the frequency tuning was achieved in CPW resonators by inserting a superconducting quantum interference device (SQUID) into the resonator as a control element. Such a scheme allows for fast $(\sim 1 \mathrm{~ns})$ and wide range $(\sim 700 \mathrm{MHz})$

a)Electronic mail: astghik@chalmers.se tuning but at the expense of introducing an extra dissipation channel associated with the SQUID. This limits the quality factor to maximum $\sim 10^{4}$ at the original frequency and $<2000$ -3000 for the maximum detuning. ${ }^{17}$

As an alternative to SQUIDs, tuning the resonance frequency can be accomplished by controlling the kinetic inductance of the superconducting line itself. This approach allows to preserve the high quality factor of the original resonator (up to $10^{6}$ for CPW on sapphire) and does not require dilution fridge temperatures for SQUID operation. Design wise, the simplest way to adjust the kinetic inductance of a superconductor is by applying external magnetic field. This solution, originally suggested in Ref. 18, however provides only limited tuning $<3 \mathrm{MHz}$ and essentially DC control on resonance frequency. To achieve a dynamic tuning one can exploit the current-dependent nonlinearity of kinetic inductance, as suggested in Ref. 19. Practically, introducing a current bias into a microwave line is not a trivial task, as control lines should not drain too much microwave energy from the resonator. ${ }^{20,21}$ This implies some trade-off between tuning time and radiation losses. While maintaining a high internal quality factor ${ }^{22} \sim 2 \times 10^{5}$, the current-directing circuit suggested in Ref. 19 constrained the tuning time well below $\sim 1 \mathrm{~ns}$ achievable with SQUIDS. ${ }^{17}$

In this work, we present fabrication and low temperature study of microstrip resonators deploying an atomic layer deposited (ALD) aluminum oxide as the dielectric material. We report a loss tangent in a thin $(\sim 50 \mathrm{~nm})$ dielectric film as low as $\tan \delta \sim 10^{-4}$ when the resonator is loaded with a single photon; at elevated powers, losses rapidly drop below $\tan \delta \sim 10^{-5}$ for $\sim 50$ photons in the resonator. Exploiting the versatility of the microstrip design, we implement a straightforward extension of the basic design incorporating a current control over the strip kinetic inductance. The resulting design combines conceptual simplicity, wide tuning range $(\sim 60 \mathrm{MHz})$, fast tuning time $(\sim 3 \mathrm{~ns})$, and a quality factor on par $\left(10^{4}\right.$ for single photon load) or superior (at an increased power) to common CPW/SQUID solution. 
The microstrip resonators are implemented as a multilayer $\mathrm{Nb} / \mathrm{Al} / \mathrm{Al}_{2} \mathrm{O}_{3} / \mathrm{NbN}$ structure on a 2 in. sapphire wafer. The bottom $\mathrm{Nb}$ layer serves as the ground plane conductor of the microstrip line; the coplanar excitation line was also patterned in this layer. The top conductor of the microstrip line and coupling capacitors were defined in the top $\mathrm{NbN}$ layer. The thin Al layer is a seeding layer which initiates a high quality ALD aluminum oxide in the subsequent step. During fabrication, first $50 \mathrm{~nm}$ of $\mathrm{Nb}$ was sputtered over the wafer, followed by $10 \mathrm{~nm}$ of $\mathrm{Al}$ in the same near-UHV sputtering system. Right after, the chamber was filled with an oxygen at 6.5 Torr and the sample was oxidized for $10 \mathrm{~min}$ at $170^{\circ} \mathrm{C}$, resulting in $\sim 5 \mathrm{~nm}$ of $\mathrm{Al}_{2} \mathrm{O}_{3}$. The coplanar line was defined in the $\mathrm{Nb} / \mathrm{Al} / \mathrm{Al}_{2} \mathrm{O}_{3}$ layer with standard e-beam lithography and reactive ion etching (RIE). Next, $50 \mathrm{~nm}$ of ALD aluminum oxide was deposited. To achieve a high quality of ALD dielectric, we followed the recipe developed in Ref. 23. Finally, the top $50 \mathrm{~nm} \mathrm{NbN}$ layer was sputtered and patterned with e-beam and RIE etching.

To characterize the quality of ALD oxide layer, we first fabricated basic $\lambda / 2$ microstrip resonators capacitively coupled to a CPW feedline, as shown in Fig. 1(a), and measured the device performance in a $\mathrm{He}^{3}$ cryostat with a base temperature $\mathrm{T}=0.3 \mathrm{~K}$.

The device transmission was measured with a vector network analyzer (VNA) and the resonance line shape was fitted to a Lorentzian ${ }^{24}$

(a)

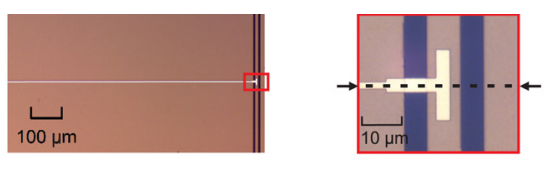

(b)

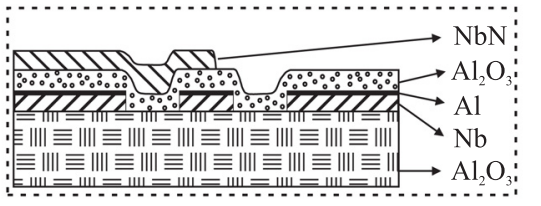

(c)

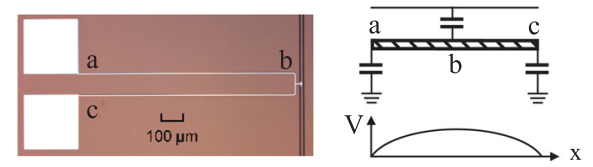

(d)

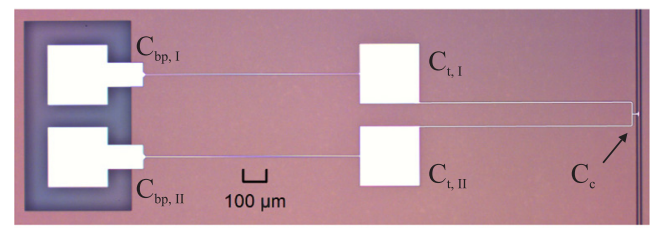

(e)

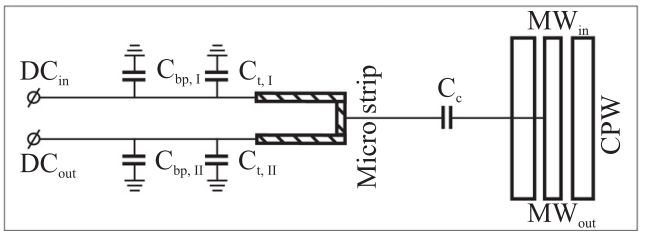

FIG. 1. (a) Optical microscope (OM) image of a basic $\lambda / 2$ microstrip resonator capacitively coupled to a CPW feedline and zoom-in on the coupling area. (b) A sketch of the structure cross-section across the dotted line in (a)-right, not to scale. (c) Left: OM image of a $\lambda / 2$ resonator terminated with shunt capacitors in both ends and coupled in the middle point. Right: schematic representation and the microwave voltage mode structure. (d) Same structure as in (c), but with shunt capacitors connected to bonding pads. (e) Schematic representation for the design in (d).

$$
S_{21}=1+S_{21, b c k g}-\left(1-S_{21, \text { min }}\right) /(1+2 i Q \delta f),
$$

where $f_{0}$ is the resonance frequency, $\delta f=\left(f-f_{0}\right) / f_{0}$ is the normalized frequency detuning, $S_{21, \min }=Q_{c} /\left(Q_{i}+Q_{c}\right)$ is the minimum transmission at resonance, $Q_{i}$ and $Q_{c}$ are internal (dissipation) and external (coupling) quality factors, and the $S_{21, b c k g}$ is a complex parameter which accounts for a skewed Fano-type resonance line shape due to some residual background transmission. ${ }^{25}$

The temperature dependence of the dissipative quality factors $Q_{i}$ for two resonators measured is presented in Fig. 2(a).

The observed temperature dependence is typical and indicates that below $\sim 1 \mathrm{~K}$ the Q-factor is limited by two level fluctuators (TLFs). ${ }^{26,27}$ This conclusion is further supported by the characteristic power dependence of the quality factor at the base temperature ${ }^{28,29}$ presented in Fig. 2(b). For an excitation power $P$, the energy stored in the resonator is given by $E=2\left(Z_{0} / Z_{R}\right) Q_{c} P / 2 \pi f_{0}$ (for $Q_{c} \ll Q_{i}$ ); having the wave impedances of the excitation line $Z_{0}=50 \Omega$, the resonator $Z_{R}=26 \Omega$ (see below), and $Q_{c} \sim 250$ known from the fit, we see that at $-130 \mathrm{dBm}$, the resonators are loaded with 2-3 photons. For a microstrip line with a width $w=2 \mu \mathrm{m}$ much higher than a dielectric thickness $d=50 \mathrm{~nm}$, the loss tangent is simply $\tan \delta=1 / Q_{i} \sim 10^{-4}$. The observed loss
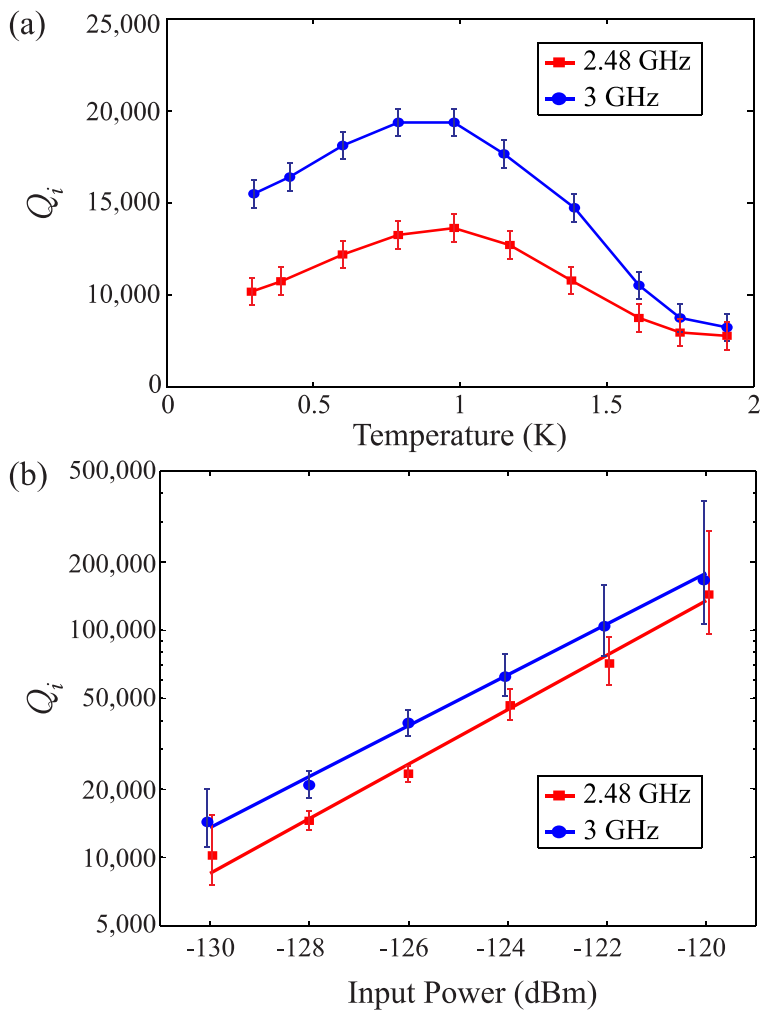

FIG. 2. Losses in basic $\lambda / 2$ microstrip resonators. (a) The temperature dependence of the internal quality factor $Q_{i}$ for two devices measured. Measurements were performed at an excitation power $-130 \mathrm{dBm}$. The dots are the measurements and the lines are guides for the eye. (b) The power dependence of $Q_{i}$ measured at a base temperature $T=0.3 \mathrm{~K}$. The dots represent the data and the lines are best linear fits. To calibrate the excitation power, all components in the input line were separately characterized in a gas flow cryostat at $2 \mathrm{~K}$. Our estimate for a maximum power calibration error is $3 \mathrm{~dB}$. At still higher input powers, $Q_{i}$ cannot be extracted from the fit to Eq. (1), because the fitting procedure returns $Q_{i}$ with an uncertainty approaching $100 \%$. 
tangent is on par with $2.7 \times 10^{-5}$ previously reported for ALD $\mathrm{Al}_{2} \mathrm{O}_{3}{ }^{30}$

To add a frequency-tuning functionality, we choose to follow the design concept suggested in Ref. 31: to terminate a segment of the microwave line on both sides with large shunt capacitors. These capacitors effectively provide shorts to the ground at resonance frequency while, at the same time, they operate as low-pass filters transmissive for current bias control (a detailed description follows). We note that, in contrast to Ref. 31, for a microstrip design, no extra fabrication step is needed to incorporate terminal capacitors.

To verify that the large area $\left(250 \times 250 \mu \mathrm{m}^{2}\right)$ terminal capacitors do not spoil the resonance quality, we fabricated the design shown in Fig. 1(c): a $\lambda / 2$ resonator with two voltage nodes set by terminal shunt capacitors and capacitive coupling in the middle point. The device was characterized at $2 \mathrm{~K}$ and we measured $Q_{i}=7300$, in accordance with expectations: as the shunt capacitors are placed in voltage nodes, they should not dissipate much energy. Indeed, dielectric losses in a capacitor can be represented as an equivalent serial resistance $R_{E S R}=\tan \delta /\left(2 \pi f_{0} C_{t}\right)$, the corresponding quality factor being $Q_{E S R}=Z_{R} 2 \pi f_{0} C_{t} / \tan \delta$. As the terminal capacitors in our design have $C_{t}=110 \mathrm{pF}$, we see that $Q_{E S R}$ is negligible as soon as $\tan \delta<10^{-3}$.

Next, we made a design where terminal capacitors are wired to bonding pads, as shown in Fig. 1(d). The bonding pads are placed on top of a window opened in the ground plane to avoid possible shorts through ALD dielectric because of the bonding (as a result, bonding pads have negligible capacitance to the ground: $\left.C_{b p, I / I I} \ll C_{t}\right)$. Fig. 3 presents the effect of the DC current bias through the microstrip line: a set of transmission resonances $S_{21}$ measured for
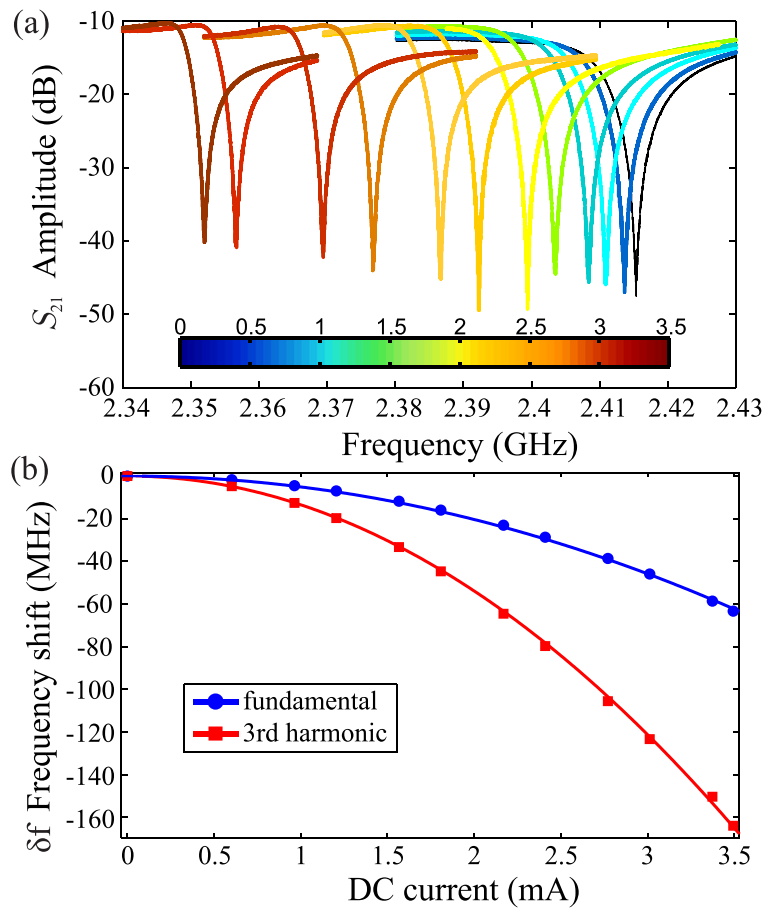

FIG. 3. Characterization of a tunable resonator performance at $0.3 \mathrm{~K}$. (a) $S_{21}$ transmission plots taken at different DC bias control currents. (b) Frequency shift vs. DC current for the fundamental frequency $2.4 \mathrm{GHz}$ and the third harmonic $7.2 \mathrm{GHz}$. The solid lines are quadratic fits to the data. different biases (a) and the resonance frequency shift vs. bias for the fundamental mode and the third harmonic (b).

Wiring to the control lines introduced some minor radiation losses so that the internal Q-factor was found to be $Q_{i}=5700$ (5800) for the first (third) harmonic. These losses are presumably due to increased coupling to background resonances (via DC lines), which is also revealed in the slightly distorted Fano-type resonance line shapes and frequencydependent $S_{21, \text { min }}$ as seen in Fig. 3.

As expected, the frequency shift in Fig. 3(b) nicely follows a parabola, as the kinetic inductance of a superconductor is a quadratic function of the bias current: ${ }^{32}$

$$
L_{K}(I) \approx L_{K}(0)\left(1+\left(I / I_{*}\right)^{2}\right),
$$

where $I$ is the bias current and $I_{*}$ is the non-linearity parameter. If the kinetic inductance comprises the dominant part of the total inductance (which is the case for a $50 \mathrm{~nm} \mathrm{NbN}$ film ${ }^{23}$ ), then

$$
\delta f / f_{0}=\sqrt{L_{K} C_{R}} \delta\left(1 / \sqrt{L_{K} C_{R}}\right) \sim-1 / 2\left(I / I_{*}\right)^{2},
$$

and the nonlinearity can be extracted from the fit: $I_{*}$ $=16 \mathrm{~mA}$, on par with that reported for a similar $\mathrm{NbN}$ film in Ref. 33.

For further discussion, it is instructive first to estimate the basic parameters of our microstrip design. The total capacitance can be deduced from the resonator dimensions (for a $2.4 \mathrm{GHz}$ device): the length $L=2280 \mu \mathrm{m}$ and the width $w=2 \mu \mathrm{m}$, assuming $\epsilon=10$, translate into $C_{R}=8.1 \mathrm{pF}$. From this, we can deduce the resonator inductance $L_{R}=1$ / $\left(4 f_{0}^{2} C_{R}\right)=5.4 \mathrm{nH}$, the characteristic line impedance $Z_{R}$ $=\sqrt{L_{R} / C_{R}}=26 \Omega$, and the phase velocity $\nu_{p h}=L / \sqrt{L_{R} C_{R}}$ $=0.038 c$ (with $c$ as the speed of light). We see that the phase velocity is $\sim 3$ times lower than for a typical CPW design (simply because of the increased per unit length capacitance of the microstrip). This allows for a very compact resonator design, which is advantageous for sensor applications like near field scanning microwave microscope. ${ }^{34,35}$ If desired, even lower $\nu_{p h}$ can be reached by boosting kinetic inductance with a thinner film ( $\mathrm{NbN}$ is superconducting from 2 to $3 \mathrm{~nm})^{36}$ or, ultimately, turning to $\mathrm{InO}_{\mathrm{x}}$ as a superconducting material. ${ }^{37}$ An extremely low phase velocity (together with a possibility of fast tuning) is beneficial for experiments on the dynamical Casimir effect ${ }^{14}$ and may allow for the observation of similar effects predicted, but not yet demonstrated, like the Hawking radiation. ${ }^{38}$

We note also that apart from being squeezed in the longitudinal direction, the microwave field volume is also cramped more than ten times in the transversal direction (from a few microns gap in CPW down to $50 \mathrm{~nm}$ gap in microstrip). As mentioned earlier, this translates into a boosted coupling to qubits and spins. Varying the dielectric thickness and/or the strip kinetic inductance (via superconductor thickness), the characteristic impedance can be custom tuned in very wide limits, thus optimizing for either magnetic or electric coupling. In terms of Q-factor, while $10^{4}$ is sufficient for many applications, we foresee that it can be further improved 2-5 times by switching to a hydrogen rich amorphous silicon (a-SiH), where a loss tangent $\approx 2 \times 10^{-5}$ was reported in a 
single-photon regime. ${ }^{29,39}$ If operation at a single-photon level is not actually needed (for tunable filters), then $Q \sim 10^{5}$ is competitive to CPWs.

Next, we turn to the tuning time. Fundamentally, the tuning time for a control current is only limited by a propagation time $1 / f_{0}$. In practice, however, the control current should be injected via some low pass filter to avoid microwave energy leakage through the control lines. In a previously reported solution, ${ }^{19}$ such a filter was composed of a $r_{F}=0.04 \Omega$ resistor inserted into excitation line and $l_{F}=2 \mathrm{nH}$ choke inductance, thus limiting the control time to $\tau_{C}$ $=l_{F} / r_{F}=50 \mathrm{~ns}=220 f_{0}^{-1}$. As the internal $Q_{i}$ was limited by losses in dissipative $r_{F}$, a high $l_{K}$ was needed to effectively isolate $r_{F}$, and $r_{F}$ had to be kept small; both requirements slow down the response time. In our design, we make use of the simplest non-dissipative low pass filter: a capacitor. For the resonance frequency, it provides a residual transmission $T \approx 1 / 2 \pi f_{0} Z_{R} C_{t} \approx 1 / 50$ (amplitude), which translates to the radiation Q-factor $1 / T^{2}=2500$, close to observations. The corresponding time constant (i.e., tuning time) is given by $Z_{R} C_{t}=3 \mathrm{~ns}$. Radiation losses can be further suppressed with a superconducting notch filter installed right after the terminal capacitor, similar to the one presented in Ref. 20; a moderate Q-factor $(\sim 10)$ band-rejection filter shall provide an extra $10 \mathrm{~dB}$ insulation for microwaves in the whole tunable range without affecting control signals.

One prospective application for the reported microstrip line would be a traveling wave parametric amplifier (TWPA). A prototype TWPA, exploiting kinetic inductance-related nonlinearity to achieve parametric amplification, was reported in Ref. 40. The achievable nonlinear frequency shift for a coplanar line used in Ref. 40 was $0.8 \%$, while in a current work we report a maximum shift of $3 \%$. Moreover, substantially reduced phase velocity in a microstrip line allows to achieve a similar parametric gain in a much shorter line, which is greatly advantageous for a practical TWPA implementation. ${ }^{33}$

Finally, we note that by applying a fractal-type perforation to the ground plane, the resonators can be made resilient to magnetic fields up to $400 \mathrm{mT}{ }^{4}$ We foresee that, by shrinking the ground plane down to a strip with the same width as the microstrip itself, operation in fields as high as $6 \mathrm{~T}$ should be feasible. ${ }^{41}$

To conclude, the microstrip resonators present a valuable design alternative to coplanar resonators; in particular, when the fast frequency tuning is a primary design goal, the achievable parameters are on par or superior to CPW-based designs. Specifically, we presented a microstrip resonator with a Q-factor above $10^{4}$ in a single-photon regime and above $10^{5}$ for $\sim 50$ photons in the resonator. We have demonstrated that a straightforward modification of the basic design allows for the current bias control over the kinetic inductance and the frequency tuning by $3 \%$ without a detrimental effect on the Q-factor. We argued that the tuning time, by design, is about $3 \mathrm{~ns}$ and can be further improved by routine circuit engineering. Inherently, the thin film microstrip resonators have dramatically reduced the microwave mode volume and, accordingly, an increased coupling to qubits and hybrid quantum memory systems, opening for numerous applications in the field of c-QED and quantum computing.
Devices were fabricated in Chalmers MC2 Nanofabrication Laboratory. We would like to thank Sebastian de Graaf and Samuel Lara Avila for technical support. We are grateful to Marie Curie Initial Training Action (ITN) Q-NET 264034 and Linne 5920344 for financial support.

${ }^{1}$ A. Wallraff, D. I. Schuster, A. Blais, R.-S. Frunzio, J. Majer, S. Kumar, S. M. Girvin, and R. J. Schoelkopf, Nature 431, 162 (2004).

${ }^{2}$ Y. Kubo, F. R. Ong, P. Bertet, D. Vion, V. Jacques, D. Zheng, A. Dreau, J. F. Roch, A. Auffeves, F. Jelezko, J. Wrachtrup, M. F. Barthe, P. Bergonzo, and D. Esteve, Phys. Rev. Lett. 105, 140502 (2010).

${ }^{3}$ D. I. Schuster, A. P. Sears, E. Ginossar, L. DiCarlo, L. Frunzio, J. J. L. Morton, H. Wu, G. A. D. Briggs, B. B. Buckley, D. D. Awschalom, and R. J. Schoelkopf, Phys. Rev. Lett. 105, 140501 (2010).

${ }^{4}$ S. E. de Graaf, A. V. Danilov, A. Adamyan, T. Bauch, and S. E. Kubatkin, J. Appl. Phys. 112, 123905 (2012).

${ }^{5}$ P. Day, H. G. LeDuc, B. A. Mazin, A. Vayonakis, and J. Zmuidzinas, Nature (London) 425, 817 (2003).

${ }^{6}$ J. Y. Mutus, T. C. White, E. Jeffrey, D. Sank, R. Barends, J. Bochmann, Y. Chen, B. Chiaro, A. Dunsworth, J. Kelly, A. Megrant, C. Neill, P. J. J. OMalley, P. Roushan, A. Vainsencher, J. Wenner, I. Siddiqi, R. Vijay, A. N. Cleland, and J. M. Martinis, Appl. Phys. Lett. 103, 122602 (2013).

${ }^{7}$ M. Devoret, S. Girvin, and R. Schoelkopf, Ann. Phys. 16, 767 (2007).

${ }^{8}$ S. E. de Graaf, J. Leppäkangas, A. Adamyan, A. V. Danilov, T. Lindström, M. Fogelström, T. Bauch, G. Johansson, and S. E. Kubatkin, Phys. Rev. Lett. 111, 137002 (2013).

${ }^{9}$ L. Athukorala and D. Budimir, IEEE Microwave Wireless Compon. Lett. 19, 698 (2009).

${ }^{10}$ K. C. Lee, H. T. Su, and M. K. Haldar, Int. J. Electron. Commun. 69, 1533 (2015).

${ }^{11}$ J. Zmuidzinas, Annu. Rev. Condens. Matter Phys. 3, 169 (2012).

${ }^{12}$ M. Sandberg, F. Persson, I. C. Hoi, C. M. Wilson, and P. Delsing, Phys. Scr. 2009, 014018.

${ }^{13}$ M. Pierre, I. M. Svensson, S. R. Sathyamoorthy, G. Johansson, and P. Delsing, Appl. Phys. Lett. 104, 232604 (2014).

${ }^{14}$ J. R. Johansson, G. Johansson, C. M. Wilson, and F. Nori, Phys. Rev. Lett. 103, 147003 (2009).

${ }^{15}$ M. A. Castellanos-Beltran and K. W. Lehnert, Appl. Phys. Lett. 91, 083509 (2007).

${ }^{16}$ M. Simoen, C. W. S. Chang, P. Krantz, J. Bylander, W. Wustmann, V. Shumeiko, P. Delsing, and C. M. Wilson, J. Appl. Phys. 118, 154501 (2015).

${ }^{17}$ Z. L. Wang, Y. P. Zhong, L. J. He, H. Wang, J. M. Martinis, and A. N. Cleland, Appl. Phys. Lett. 102, 163503 (2013).

${ }^{18}$ J. E. Healey, T. Lindström, M. S. Colclough, C. M. Muirhead, and A. Y. Tzalenchuk, Appl. Phys. Lett. 93, 043513 (2008).

${ }^{19}$ M. R. Vissers, J. Hubmayr, M. Sandberg, S. Chaudhuri, C. Bockstiegel, and J. Gao, Appl. Phys. Lett. 107, 062601 (2015).

${ }^{20}$ S.-X. Li and J. B. Kycia, Appl. Phys. Lett. 102, 242601 (2013).

${ }^{21}$ S. E. de Graaf, D. Davidovikj, A. Adamyan, S. Kubatkin, and A. Danilov, Appl. Phys. Lett. 104, 052601 (2014).

${ }^{22}$ The excitation power was not specified in the reference.

${ }^{23}$ A. A. Adamyan, S. E. de Graaf, S. E. Kubatkin, and A. V. Danilov, Supercond. Sci. Technol. 28, 085007 (2015).

${ }^{24}$ M. S. Khalil, M. J. A. Stoutimore, F. C. Wellstood, and K. D. Osborn, J. Appl. Phys. 111, 054510 (2012).

${ }^{25}$ The dominant contribution to background transmission normally comes from the package and can typically be modeled as a parallel capacitance. Within the resonance line width, the transmission through this capacitance can be represented by some complex constant and results in an asymmetric resonance line shape. The background transmission is typically rather small, but could be noticeable if the (tunable) resonator frequency matches the frequency of some ground plane resonance on a chip.

${ }^{26}$ P. Macha, S. H. W. van der Ploeg, G. Oelsner, E. Ilichev, H. G. Meyer, S. Wönsch, and M. Siegel, Appl. Phys. Lett. 96, 062503 (2010).

${ }^{27}$ M. Arzeo, F. Lombardi, and T. Bauch, Appl. Phys. Lett. 104, 212601 (2014).

${ }^{28}$ T. Lindström, J. E. Healey, M. S. Colclough, C. M. Muirhead, and A. Y. Tzalenchuk, Phys. Rev. B 80, 132501 (2009).

${ }^{29}$ A. D. O'connell, M. Ansmann, R. C. Bialczak, M. Hofheinz, N. Katz, E. Lucero, C. McKenney, M. Neeley, H. Wang, E. M. Weig, A. N. Cleland, and J. M. Martinis, Appl. Phys. Lett. 92, 112903 (2008).

${ }^{30}$ J. Burnett, T. Lindström, M. Oxborrow, Y. Harada, Y. Sekine, P. Meeson, and A. Y. Tzalenchuk, Phys. Rev. B 87, 140501(R) (2013).

${ }^{31}$ S. J. Bosman, V. Singh, A. Bruno, and G. A. Steele, Appl. Phys. Lett. 107, 192602 (2015). 
${ }^{32}$ R. W. Landauer, U.S. patent 3,111,628 (19 November 1963).

${ }^{33}$ A. A. Adamyan, S. E. de Graaf, S. E. Kubatkin, and A. V. Danilov, J. Appl. Phys. 119, 083901 (2016).

${ }^{34}$ S. E. de Graaf, A. V. Danilov, A. Adamyan, and S. E. Kubatkin, Rev. Sci. Instrum. 84, 023706 (2013).

${ }^{35}$ S. E. de Graaf, A. V. Danilov, and S. E. Kubatkin, Sci. Rep. 5, 17176 (2015).

${ }^{36}$ J. T. Peltonen, O. V. Astafiev, Y. P. Korneeva, B. M. Voronov, A. A. Korneev, I. M. Charaev, A. V. Semenov, G. N. Goltsman, L. B. Ioffe, T. M. Klapwijk, and J. S. Tsai, Phys. Rev. B 88, 220506(R) (2013).
${ }^{37}$ O. V. Astafiev, L. B. Ioffe, S. Kafanov, Y. A. Pashkin, and J. S. Tsai, Nature (London) 484, 355 (2012).

${ }^{38}$ R. Schützhold and G. Unruh, Phys. Rev. Lett. 95, 031301 (2005).

${ }^{39}$ B. A. Mazin, D. Sank, S. McHugh, E. A. Lucero, A. Merrill, J. Gao, D. Pappas, D. Moore, and J. Zmuidzinas, Appl. Phys. Lett. 96, 102504 (2010).

${ }^{40}$ H. B. Eom, P. K. Day, H. G. LeDuc, and J. Zmuidzinas, Nat. Phys. 8, 623 (2012).

${ }^{41}$ N. Samkharadze, A. Bruno, P. Scarlino, G. Zheng, D. P. DiVincenzo, L. DiCarlo, and L. M. K. Vandersypen, Phys. Rev. Appl. 5, 044004 (2016). 